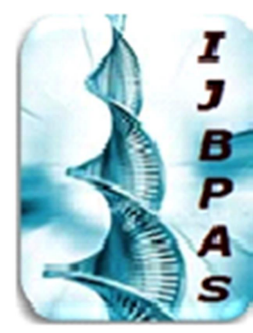

International Journal of Biology, Pharmacy and Allied Seiences (IJBPAS) 'A Bridge Betusen Caboratory and QRader'

WwW.iibpas.com

A CASE REPORT ON TUBERCULOUS SMALL BOWEL STRICTURE CAUSING SUBACUTE OBSTRUCTION

\title{
AKSHAYA $\mathbf{G}^{1^{*}}$ AND RAGUPATHY $\mathrm{T}^{2}$
}

1: Junior Resident, Department of General Surgery, Sree Balaji Medical College \& Hospital, Bharath University, Chrompet, Chennai, India

2: Professor, Department of General Surgery, Sree Balaji Medical College \& Hospital, Bharath University, Chrompet, Chennai, India

*Corresponding Author: Akshaya G: E Mail: akshaya.gk@gmail.com

Received 28 ${ }^{\text {th }}$ March 2021; Revised 28 ${ }^{\text {th }}$ April 2021; Accepted $25^{\text {th }}$ May 2021; Available online $1^{\text {st }}$ Dec. 2021 https://doi.org/10.31032/IJBPAS/2021/10.12.5798

\begin{abstract}
Tuberculosis can involve the entire gastrointestinal tract. Abdominal tuberculosis is the 6 th most common site for extra pulmonary TB. It is more common in India and developing countries. This case report describes a case of sub acute intestinal obstruction due to tuberculous intraluminal stricture of ileum.
\end{abstract}

Keywords: Tuberculosis, acute intestinal obstruction, subacute obstruction INTRODUCTION

Tuberculosis is the most common and widespread disease in India. The primary site of TB is usually lungs from which it gets disseminated. But sometimes primary could be from extra pulmonary sites. Abdominal tuberculosis is difficult to diagnose because of its vague presentation. Abdominal TB could present as Luminal gastrointestinal tuberculosis, abdominal solid organ tuberculosis, peritoneal tuberculosis and abdominal lymph node tuberculosis. Abdominal tuberculosis is more common between the age of 25 to 45 years, most common being intestinal of which ileocecal is $60 \%$ and ileal is $40 \%$. Infection results in granuloma formation, 
caseation, ulceration, fibrosis, scarring and

it could present as ulcerative, hyperplastic or stricture type. A combination of clinical signs, radiological and histopathological work up is essential for promt diagnosis and management [1-4].

\section{CASE REPORT}

A 37 year old male presented to OPD with complaints of periumblical pain since 2 months, dull aching type, intermittent, radiating to back, aggravated on food intake, associated with nausea, occasional vomiting few hours after food intake and anorexia. Able to tolerate liquids but not solids. There was a weight loss of $25 \mathrm{kgs}$ in 2 months. He had history of evening rise of temperature for 1 week. There was no history of tuberculosis or contact with tuberculosis patients. On examination his abdomen was soft, bowel sounds were present and there was tenderness in the periumblical region.

All Routine work up was done. His blood investigations did not show any abnormality other than mild elevated lymphocyte count. Mauntoux test was positive $(24 \mathrm{~mm})$. His chest $\mathrm{x}$-ray did not show any abnormality. Usg abdomen showed dilated small bowel loops. CECT Abdomen was done which showed - Long segment diffuse circumferential wall thickening with $1.1 \mathrm{~cm}$ in proximal ileal loops for a distance of $17 \mathrm{~cm}$ causing luminal narrowing. Multiple enlarged lymph nodes seen in the mesenteric region and around the wall thickening.Suggesting sub acutesmall bowel obstruction.

On exploratory laparotomy, the findings (Figure 1) were luminal narrowing in the proximal ileum with induration, proximal loop distended and distal loop collapsed with multiple mesenteric lymph nodes. Resection $23 \mathrm{~cm}$ of proximal ileum (Figure 2) was done and specimen sent for histopathology.

On gross examination stricture in the proximal end of resected bowel with loss of mucosal folds. On histopathological examination showed hyperplastic mucosa with crowded glands showing cystic dilatation and ulceration of mucosa with multiple plasma cells, admixed with eosinophils, lymphocytes and polymorphs and granuloma formation in lamina propria; thrombosed and congested serosal vessels. Lymph nodes show epitheloidgranuloma formation, langhans giant cells with caseation and changes suggestive of tuberculous small bowel stricture.

Post operative period was uneventful. Patient recovered progressively. Tolerated liquids and solids on post operative days 4 and 5. And was discharged onpost operative day 8 . Patient was started on anti tubercular treatment and during follow up patient's condition improved well. 


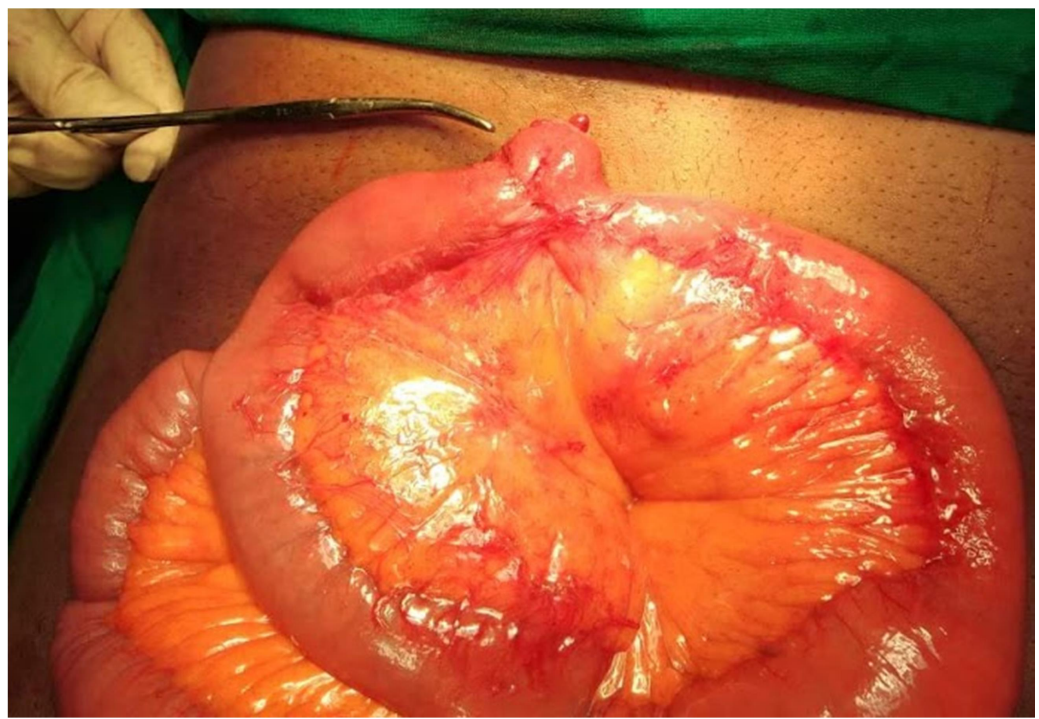

Figure 1: Proximal ileal stricture

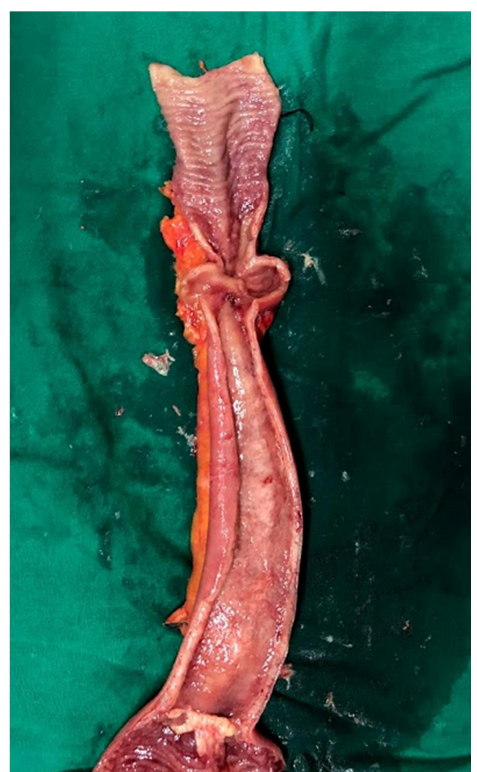

Figure 2: Cut section of the structured bowel showing loss of mucosal folds

\section{DISCUSSION}

Small bowel obstruction is most commonly caused by adhesions, hernias, neoplasms, foreign bodies and inflammatory strictures. Among strictures the most common causes are tuberculosis, crohn's disease, typhoid and NSAID use [1].

Abdominal TB is one the most prevalent forms. Types of abdominal TB include intestinal type (ulcerative $-60 \%$, Hyperplastic, stricture $40 \%$ ), peritoneal, TB of mesentry, anorectosigmoidal, esophageal and involvement of other organs. Tuberculou bacilli spread to the GI tract through various modes of spread. Modes of spread of infection are ingestion of food/ sputum contaminated with tubercle bacilli, Hematogenous spread from lungs, From 
neck nodes through lymphatics. 2-4 weeks later it causes caseation, necrosis, ulceration - girdle ulcers and strictures napkin ring strictures, spread into deeper layer finally involving the lymph nodes leading to spread of infection. And complications which include small bowel obstruction, blind loop syndrome, fecal fistulas, cold abscess formation, haemorrhage and perforation $[1,2]$.

Abdominal tuberculosis is difficult to diagnose because of its vague presentation and no gold standard investigation. Hence diagnosis is made by combined clinical, radiological and histopathological studies.From clinical symptoms and signs patient may present with vague abdominal pain, loss of weight and appetite, features of obstruction, mass in right iliac fossa. Chest $\mathrm{x}$-ray to find out primary focus; plain x-ray abdomen for features of obstruction, CT scan of abdomen showing thickened bowel wall, ileocecal valve thickening, adhesions, mesenteric nodes, obstruction and loculated ascites; serum ADA of more than 54IU, barium meal showing narrow ileum, pulled up cecum, straightening of ileocecal junction (gooseneck deformity), asictic fluid protein more than 2.5 $\mathrm{g} / \mathrm{dl}$,demonstration of acid fast bacilli and finally caseation necrosis in hispathology is the hallmark in diagnosing tuberculosis.

Treatment of abdominal tuberculosis involves starting of anti tubercular therapy and supportive treatment for malnourishment. Surgery is required in cases of intestinal obstruction, perforation, intraabdominalabscess formation and haemorrhage. Surgeries could be of three types first being bypassing the involved segment of bowel like ostomy, second being resection and anastomosis, and third being simple stricteroplasty. Limitations of surgical management are malnourished status of the patient and widely places lesions making them poor candidates for surgery $[2,3]$.

In this case report patient presented with vague symptoms with diagnosis towards carcinoma. Only on further evaluation and surgery patient was diagnosed to have tuberculosis which is a life threatening disease. The diagnosis of extra pulmonary TB could be difficult because of its nonspecific presentation and requires high degree of suspicion for diagnosis.

\section{CONCLUSION}

Tuberculosis- pulmonary or extrapulmonary, a global burden because of its wide spread nature, patients should be actively investigated, diagnosed and treated promptly with proper patient education.

\section{REFERENCE}

[1] Lazarus AA, Thilagar B. Abdominal tuberculosis. Disease-aMonth. 2007 Jan 1; 53(1): 32-8.

[2] George Van Buren II, Teichgraeber DC, Ghorbani RP, Souchon EA. 
Sequential stenotic strictures of the small bowel leading to obstruction.

World Journal of Gastroenterology:

WJG. 2007 Oct 28; 13(40): 5391.

[3] Smink DS. Schwartz's principles of surgery.

[4] Beauchamp DR, Evers BM, Mattox KL. Sabiston textbook of surgery: the biological basis of modern surgical practice. Elsevier Brasil; 2004. 